\title{
Community-acquired methicillin-resistant Staphylococcus aureus pneumonia in a children's hospital. Our ten-year experience
}

\author{
Gabriela Ensinck, M.D. ${ }^{a}$, Gustavo Lazarte, M.D. ${ }^{a}$, Adriana Ernst, Biochemist, ${ }^{a}$ \\ Antonela Romagnoli, M.D. ${ }^{a}$, Santiago López Papucci, M.D. ${ }^{a}$, Alicia Aletti, M.D. ${ }^{a}$, \\ Ana Chiossone, M.D. ${ }^{a}$, Fernanda Pigozzi, B.S. ${ }^{a}$ and Yanina Sguassero, M.D. ${ }^{b}$
}

\begin{abstract}
Introduction. Community-acquired methicillinresistant Staphylococcus aureus (CA-MRSA) infections have increased in recent years. CAMRSA necrotizing pneumonia and empyema are now more common in children.

Objectives. To determine the prevalence of CA-MRSA pneumonia and its clinical and epidemiological characteristics compared to Streptococcus pneumoniae (SP) pneumonia in the same population.

Materialandmethods. Descriptive, observational, cross-sectional study of patients hospitalized due to CA-MRSA pneumonia at Hospital de Niños Víctor J. Vilela (period: January 2008-December 2017).

Results. Out of 54 Staphylococcusaureus pneumonia cases, $46(85 \%)$ corresponded to CA-MRSA. The rate of CA-MRSA pneumonia ranged from 4.9/10000 (2008) to 10/10000 hospital discharges (2017). Sepsis/septic shock was observed in $41 \%$; empyema, in $96 \%$; pneumothorax, in $35 \% ; 90 \%$ of cases required pleural drainage and $55 \%$, surgical debridement. Also, $65 \%$ of patients were admitted to the intensive care unit (ICU); half of them required assisted mechanical ventilation. Two patients died. Strain resistance: $17 \%$, gentamicin; $13 \%$, erythromycin; and $11 \%$, clindamycin. Compared to SP pneumonia, CAMRSA pneumonia showed a higher risk for sepsis (95\% confidence interval; relative risk: 7.38 ; 3.3216.38 ) and admission to the ICU (RR: 4.29 ; 2.70 6.83). No patient died due to SP pneumonia.

Conclusions. The prevalence of CA-MRSA pneumonia doubled in the past decade. Compared to SP pneumonia, CA-MRSA pneumonia was more commonly accompanied by sepsis and septic shock, admission to the ICU, and ventilatory support requirement.

Key words: Staphylococcal pneumonia, methicillinresistant Staphylococcus aureus; cross-sectional studies; children's hospitals.
\end{abstract}

http: / / dx.doi.org/10.5546/ aap.2021.eng.11

To cite: Ensinck G, Lazarte G, Ernst A, Romagnoli A, et al. Community-acquired methicillin-resistant Staphylococcus aureus pneumonia in a children's hospital. Our ten-year experience. Arch Argent Pediatr 2021;119(1):11-17.

\section{INTRODUCTION}

The worldwide increase in Staphylococcus aureus (SA) infections has been mainly related to community-acquired methicillinresistant Staphylococcus aureus (CAMRSA).$^{1-3}$ These strains have a type IV or V staphylococcal cassette chromosome mec (SCCmec). This is a mobile genetic element that carries the central determinant for beta-lactam resistance encoded by the mecA gene, which confers resistance to betalactam antibiotics and may also lead to accompanying resistance to other antibiotic groups, such as macrolides and aminoglycosides. ${ }^{4-6}$ In general, they have an exotoxin called PantonValentine leukocidin (PVL), which has been associated with invasiveness, necrosis, and a poor prognosis. ${ }^{7,8}$

CA-MRSA infections usually present as skin and soft tissue infections. However, in recent years, invasive infections have increased, including complicated pneumonia, arthritis, and osteomyelitis..$^{9,10}$ Complicated pneumonia (CP) is defined as that accompanied by pleural effusion, empyema, necrotizing pneumonia or pneumonia with abscess formation. ${ }^{11}$

Staphylococcal pneumonia used to be an uncommon condition in pediatrics; however, it has become increasingly frequent and has an important morbidity and mortality. ${ }^{12}$ The primary objective of this study was to assess the prevalence of CAMRSA pneumonia in the pediatric population of our hospital and its clinical and epidemiological characteristics. A secondary objective was to compare it to the prevalence 
and characteristics of Streptococcus pneumoniae (SP) pneumonia recorded in the same population during the study period.

\section{MATERIAL AND METHODS}

This was a descriptive, prospective, crosssectional study in children and adolescents with a microbiologically documented diagnosis of CA-MRSA pneumonia assessed at Hospital de Niños Víctor J. Vilela between January 2008 and December 2017. Exclusion criteria were patients who, in the six months prior to study inclusion, had been hospitalized (at least, 24 hours), had visited a health care center or been in contact with health care staff, had received antibiotics or had a history of MRSA isolation. In addition, patients with comorbidities at the time of admission, such as immunosuppression, diabetes, corticosteroid therapy, etc. were excluded. Patients who did not have all data of analyzed outcome measures available were not excluded, but this was clarified in each case. Infection was defined as communityacquired when SA growth was isolated in at least one culture from a sterile site in outpatients or in the first 48 hours of hospitalization.

In relation to microbiological diagnosis, the culture sample was obtained from sterile sites (pleura, joint, surgical material, blood cultures, etc.). SA was isolated and typified using conventional automated microbiological tests based on the working protocols currently in place at the hospital's Microbiology Laboratory. Methicillin sensitivity was determined using the disk-diffusion method with a cefoxitin 30-microgram $(\mu \mathrm{g})$ disk; a halo smaller than 20 milliliters $(\mathrm{mm})$ was defined as methicillin resistance. Resistance to rifampicin $(5 \mu \mathrm{g})$, gentamicin $(10 \mu \mathrm{g})$, trimethoprimsulfamethoxazole $(25 \mu \mathrm{g})$, erythromycin $(15 \mu \mathrm{g})$, clindamycin $(2 \mu \mathrm{g})$, and ceftaroline $(5 \mu \mathrm{g})$ was also determined, based on international standards. Antibiograms, were interpreted according to the standards of the Clinical and Laboratory Standards Institute (CLSI, 2016) ${ }^{13}$ Inducible clindamycin resistance was identified placing the clindamycin disk at $25 \mathrm{~mm}$ from the erythromycin disk in the antibiogram (D test). The minimum inhibitory concentration (MIC) of vancomycin was established by microdilution or the agar dilution method with E-test strips.

The following data were collected in a specially-designed form: sex, age, clinical presentation, medical and/or surgical treatment, antibiotic sensitivity of the isolated microorganism, length of stay, admission to the intensive care unit (ICU), initiation of assisted mechanical ventilation (AMV), and number of deaths.

Data were analyzed in an overall manner. The SAS 9.2 software was used for statistical analyses. Categorical outcome measures were described as number and percentage. The relative risk (RR) and $95 \%$ confidence interval (CI) were also calculated for primary outcomes. Continuous outcome measures were described as median and range.

The study protocol was authorized by the Ethics Committee of the Secretariat of Public Health of the Municipality of Rosario and approved by the Teaching and Research Committee of Hospital de Niños Víctor J. Vilela. No written informed consent was requested for data use. Before including a child in the study, a member of the research team described the study objectives and descriptive nature to the parents or legal guardians and noted that participation would not affect the child's clinical management or treatment indication. The research team warranted data anonymity and confidentiality.

\section{RESULTS}

During the study period, 54 patients with SA pneumonia were eligible. Eight patients, were excluded because they met an exclusion criterion, and 46 were included and analyzed $(85 \%)$. The annual rate of CA-MRSA pneumonia in relation to hospital discharges ranged from 4.9/10 000 (2008) to 10.1/10 000 (2017) (Table 1, Figure 1). The average age of the 46 patients with CA-MRSA pneumonia was 44 months (median: 27.5 months). Out of all patients, $24(52 \%)$ were females. The average number of people sharing the household with patients was 4.5 (range: 2-8). A history of infections or an active infection at the time of diagnosis in a family member was confirmed, including impetigo, cellulitis, furunculosis or recurrent furunculosis in $37.5 \%$ of cases (Table 2).

In relation to clinical manifestations, $41 \%$ had sepsis or septic shock. The chest X-ray of $22 / 46(48 \%)$ patients showed a pattern compatible with necrotizing pneumonia or pneumonia with abscess formation, and 21/46 $(46 \%)$, with consolidated or lobar pneumonia. Complications included empyema in $96 \%$ $(41 / 46)$ and pneumothorax in $35 \%$ (16/46). Skin manifestations were observed in 12 patients (26\%): 6 had scarlatiniform rash and 6, cellulitis.

A pleural drainage tube was required in 
$89 \%$ of patients; 1 tube: 19 patients; 2 tubes: 14 patients; and 3 or more tubes: 8 patients. The average length of tube placement was 16 days (range: 1-63 days). Surgical debridement was performed in more than half of patients $(25 / 46)$; in general, it was a single procedure $(75 \%)$.

The total length of stay ranged from 1 to 77 days; the median value was 24 days. Blood cultures were positive in $10 / 46$ patients $(22 \%)$. Almost $90 \%$ of pleural fluid cultures from 42 samples were positive; pleural biopsy cultures were positive in $7 / 11$ patients $(63 \%)$. Skin and soft tissue cultures were positive in $5 / 6$ patients; joint fluid cultures, in 2/3; and pericardial fluid cultures, in $2 / 4$.

The antibiogram showed that $17 \%$ of strains were resistant to gentamicin; $13 \%$, to erythromycin; $11 \%$, to clindamycin; and $2 \%$, to trimethoprim-sulfamethoxazole. No resistance to teicoplanin, vancomycin, minocycline or linezolid was observed. Sensitivity to ceftaroline was tested in 17 strains; 1 of these $(5.7 \%)$ showed intermediate sensitivity. The MIC of vancomycin was established in 45 samples; in approximately $90 \%$ of strains, it was $\leq 1 \mu \mathrm{g} / \mathrm{mL}$ (Table 3 ).

In relation to clinical course, $30 / 46$ patients $(65 \%)$ were admitted to the pediatric intensive care unit (PICU). In this subgroup, age ranged from 1 to 132 months, with a median of 23 months. In average, these patients stayed at the PICU for 9 days (maximum: 52 days). Half of patients admitted to the PICU $(15 / 30)$ required AMV, for an average of 11.4 days. Two patients $(4.34 \%)$ in this group died from sepsis and septic shock.

\section{DISCUSSION}

In recent years, the increase in CA-MRSA infections has led to a 3-4-fold increase in medical consultations due to this type of condition;

TABLE 1. Annual rate of community-acquired methicillin-resistant Staphylococcus aureus pneumonia in relation to hospital discharges. Hospital de Niños Victor J. Vilela, Rosario. 2008-2017 period

\begin{tabular}{lcccccccccc}
\hline Year & $\mathbf{2 0 0 8}$ & $\mathbf{2 0 0 9}$ & $\mathbf{2 0 1 0}$ & $\mathbf{2 0 1 1}$ & $\mathbf{2 0 1 2}$ & $\mathbf{2 0 1 3}$ & $\mathbf{2 0 1 4}$ & $\mathbf{2 0 1 5}$ & $\mathbf{2 0 1 6}$ & $\mathbf{2 0 1 7}$ \\
\hline Discharges & 8162 & 7323 & 7555 & 7816 & 7773 & 6519 & 7243 & 7380 & 6740 & 6949 \\
CA-MRSA & & & & & & & & & & \\
pneumonia & 4 & 2 & 4 & 3 & 3 & 7 & 6 & 4 & 6 & 7 \\
Rate per 10000 & 4.9 & 2.7 & 5.3 & 3.8 & 3.9 & 10.7 & 8.3 & 5.4 & 8.9 & 10.1 \\
\hline
\end{tabular}

CA-MRSA: community-acquired methicillin-resistant Staphylococcus aureus pneumonia.

Figure 1. Annual rate of staphylococcal pneumonia per 10000 discharges. Hospital de Niños Victor J. Vilela. 2008-2017 period

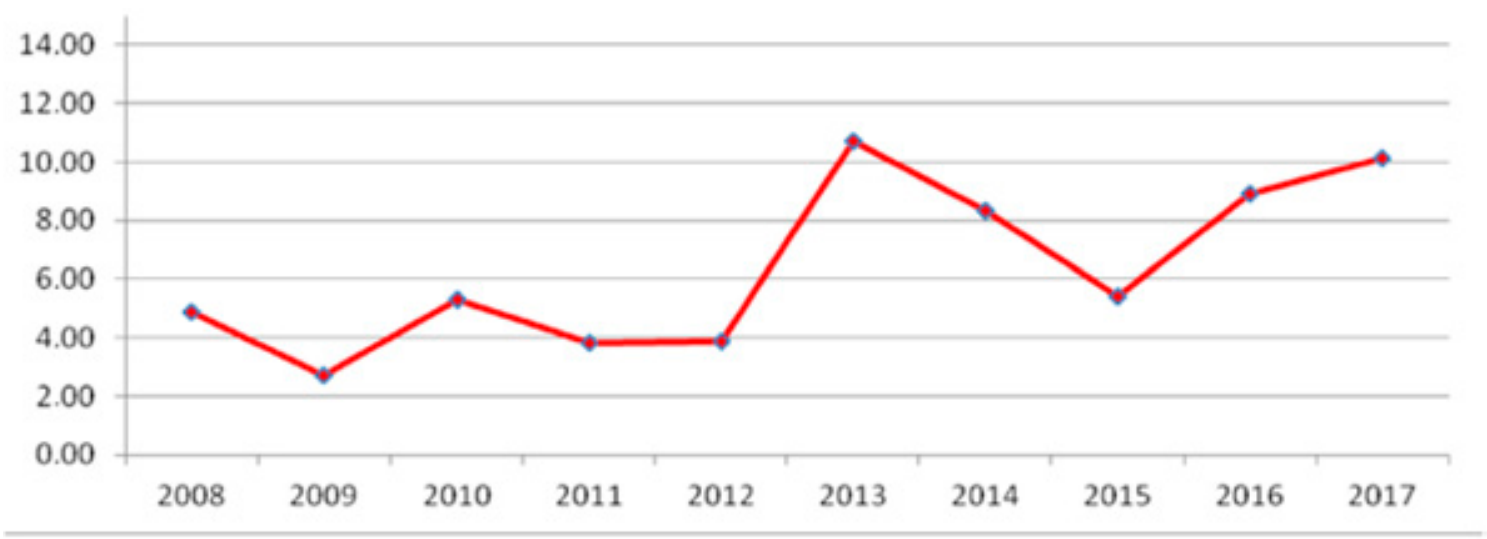

When CA-MRSA pneumonia was compared to SP pneumonia, the number of the latter decreased and the former increased (Figure 2) during the study period. Compared to SP pneumonia, patients with CA-MRSA pneumonia had a 7-fold higher risk for sepsis or septic shock, had 4 times more chances of developing necrotizing pneumonia or pneumonia with abscess formation, had 2 times more chances of having empyema, were 4 times more likely to be admitted to the PICU, and had a 5-fold higher chance of requiring AMV (Table 4). Also, unlike CA-MRSA pneumonia, no deaths were recorded in the SP pneumonia group. 
observed infections have been mild, like skin and soft tissue infections, or invasive and severe, such as sepsis, septic shock, bacteremia, osteoarticular infections, and complicated pneumonia. ${ }^{14,15}$ In a previous 10-year experience at our hospital, community-acquired SA infections were observed to increase approximately threefold, with an average $73 \%$ of CA-MRSA infections.

In this study, CA-MRSA pneumonia or empyema accounted for one of the main clinical presentations and the third leading presentation after skin and soft tissue infections and osteoarticular infections. ${ }^{16,17}$ In turn, other international publications have pointed out an increased incidence of CA-MRSA pneumonia, especially in the form of complicated pneumonia ${ }^{11,12,18}$ and mainly during the flu or influenza season..$^{19}$ Such aspect was not analyzed in our study.

During the study period, the prevalence rate of CA-MRSA pneumonia doubled in relation to hospital discharges; and such increase was mainly related to CA-MRSA, which accounts for $85 \%$ of strains. Studies conducted in Argentina in
2012-2014 described the clones circulating in the pediatric population; the most common one was ST5-IV-PVL ${ }^{+20,21}$ The average age of patients was close to 4 years; however, the median age was 27.5 months, similar to what has been reported by other authors. ${ }^{18,22}$

Different authors demonstrated that the socioeconomic level of the affected population may have an impact on the development of these infections, so this is an aspect related to poverty and overcrowding, as well as the biological

TABLE 3. Minimum inhibitory concentrations of vancomycin in community-acquired methicillin-resistant Staphylococcus aureus isolated strains. Hospital de Niños Victor J. Vilela, Rosario. 2008-2017 period

\begin{tabular}{lcc}
\hline MIC $(\mu \mathrm{g} / \mathrm{mL})$ & Number & Percentage \\
\hline 0.5 & 19 & 42 \\
0.75 & 1 & 2 \\
1 & 20 & 44 \\
1.5 & 5 & 11 \\
\hline
\end{tabular}

MIC: minimum inhibitory concentration.

TABLE 2. Clinical and epidemiological characteristics of children with community-acquired methicillin-resistant Staphylococcus aureus pneumonia $(n=46)$ and pneumococcal pneumonia $(n=118)$. Hospital de Niños Victor J. Vilela, Rosario. 2008-2017 period

\begin{tabular}{|c|c|c|c|c|}
\hline OUTCOME MEASURES & $\begin{array}{c}\text { CA-MRSA } \\
\mathrm{n}=46\end{array}$ & $\%$ & $\begin{array}{l}\text { S. pneumoniae } \\
\mathbf{n}=125\end{array}$ & $\%$ \\
\hline FEMALE & 24 & 52.2 & 56 & 45 \\
\hline $\begin{array}{l}\text { AGE (MONTHS OLD) } \\
\text { Average } \\
\text { Range } \\
\text { Median }\end{array}$ & $\begin{array}{c}44 \\
1-152 \\
27.5\end{array}$ & & $\begin{array}{c}30 \\
1-168 \\
25\end{array}$ & \\
\hline $\begin{array}{l}\text { CLINICAL PRESENTATION* } \\
\text { Consolidated pneumonia } \\
\text { Necrotizing pneumonia or } \\
\text { pneumonia with abscess formation } \\
\text { Sepsis/ septic shock } \\
\text { Pneumothorax } \\
\text { Pleural empyema } \\
\text { Skin manifestations }\end{array}$ & $\begin{array}{l}21 \\
\\
22 \\
19 \\
16 \\
44 \\
12\end{array}$ & $\begin{array}{c}45.65 \\
\\
47.8 \\
41.30 \\
34.78 \\
95.6 \\
26\end{array}$ & $\begin{array}{c}104 \\
\\
12 \\
7 \\
1 \\
52 \\
0\end{array}$ & $\begin{array}{c}9.6 \\
5.6 \\
0.8 \\
41.6 \\
0\end{array}$ \\
\hline $\begin{array}{l}\text { COURSE } \\
\text { Admission to the PICU } \\
\text { Age of patients at the PICU } \\
\text { Average length of stay at the } \text { PICU }^{2} \\
\text { AMV } \\
\text { Average days of AMV }{ }^{2} \\
\text { Pleural drainage tubes } \\
\text { Surgical debridement }\end{array}$ & $\begin{array}{c}30 \\
37.7(1-132) \\
9.4(1-52) \\
15 \\
11.4(1-43) \\
41 \\
25\end{array}$ & $\begin{array}{c}32.6 \\
89.13 \\
54.3\end{array}$ & $\begin{array}{c}19 \\
31.2(1-119) \\
6.2(1-22) \\
7 \\
7.7(1-22) \\
48 \\
14\end{array}$ & $\begin{array}{l}38.4 \\
11.2\end{array}$ \\
\hline
\end{tabular}

* The same patient had more than one clinical presentation.

${ }^{1}$ Expressed in months and range.

${ }^{2}$ Expressed in days and range.

CA-MRSA: community-acquired methicillin-resistant Staphylococcus aureus pneumonia;

PICU: pediatric intensive care unit; AMV: assisted mechanical ventilation. 
factors of the host. ${ }^{22,23}$ In this study, although the population's socioeconomic status was not considered, the number of household members was analyzed, which was, in average, equivalent to more than 4 people per household. It was also observed that almost $40 \%$ of cases had a family member with a history of infection or an active infection at the time of diagnosis. Such infection transmission phenomenon, once described in contact sports and prisoners, ${ }^{3}$ in our population may be related to poverty, overcrowding conditions, and personal hygiene issues.

Approximately $40 \%$ of patients had a clinical course with sepsis or septic shock; 2 patients died due to this condition. Such acute course, including complicated pneumonia accompanied by systemic involvement, PICU admission, and AMV requirement, has been reported by other authors at similar rates. ${ }^{18,24}$ Different publications have proposed a correlation between the presence of necrotizing pneumonia, pneumonia with abscess formation or empyema and a SA virulence factor known as PVL. ${ }^{25,26}$ PVL is a cytotoxin that causes white blood cell lysis and tissue necrosis, which undoubtedly enables SA to produce abscesses. PVL was found in both methicillinsensitive $S$. aureus (MSSA) and MRSA, so methicillin resistance is not considered a predictor of severity. ${ }^{27}$ A meta-analysis published in recent years supported the association between PVL and skin and soft tissue lesions and disregarded the relation between PVL and invasive infections. ${ }^{28}$
Almost half of patients showed radiological characteristics of necrotizing pneumonia or pneumonia with abscess formation. Most likely, the necrotizing feature of CA-MRSA pneumonia, together with AMV requirement, is a combination that results in the onset of pneumothorax during clinical course.

Microorganism isolation was high, close to $90 \%$ in pleural fluid culture and over $20 \%$ in blood culture. These values are similar to those published by other authors. ${ }^{18}$ Such situation leads us to reflect on the importance of obtaining culture samples, especially a diagnostic pleural tap, before indicating an antibiotic therapy. Clindamycin resistance was lower than that mentioned in other publications, ${ }^{24}$ while trimethoprim-sulfamethoxazole resistance was very low. Resistance to teicoplanin, vancomycin, minocycline, linezolid or ceftaroline was not confirmed, so these antibiotics should be taken into consideration for the treatment of these infections. ${ }^{29}$

When comparing pneumococcal to staphylococcal pneumonia, both with microorganism isolation, a reduction in pneumococcal pneumonia was observed in the first place, together with an increase in staphylococcal pneumonia. Without a doubt, such observation is related to the introduction of the 13-valent pneumococcal vaccine to our immunization schedule in 2011. A similar behavior was reported by other authors. ${ }^{1,30}$

FIGURE 2. Number of staphylococcal and pneumococcal pneumonia cases per year. Hospital de Niños Víctor J. Vilela. 2008-2017 period

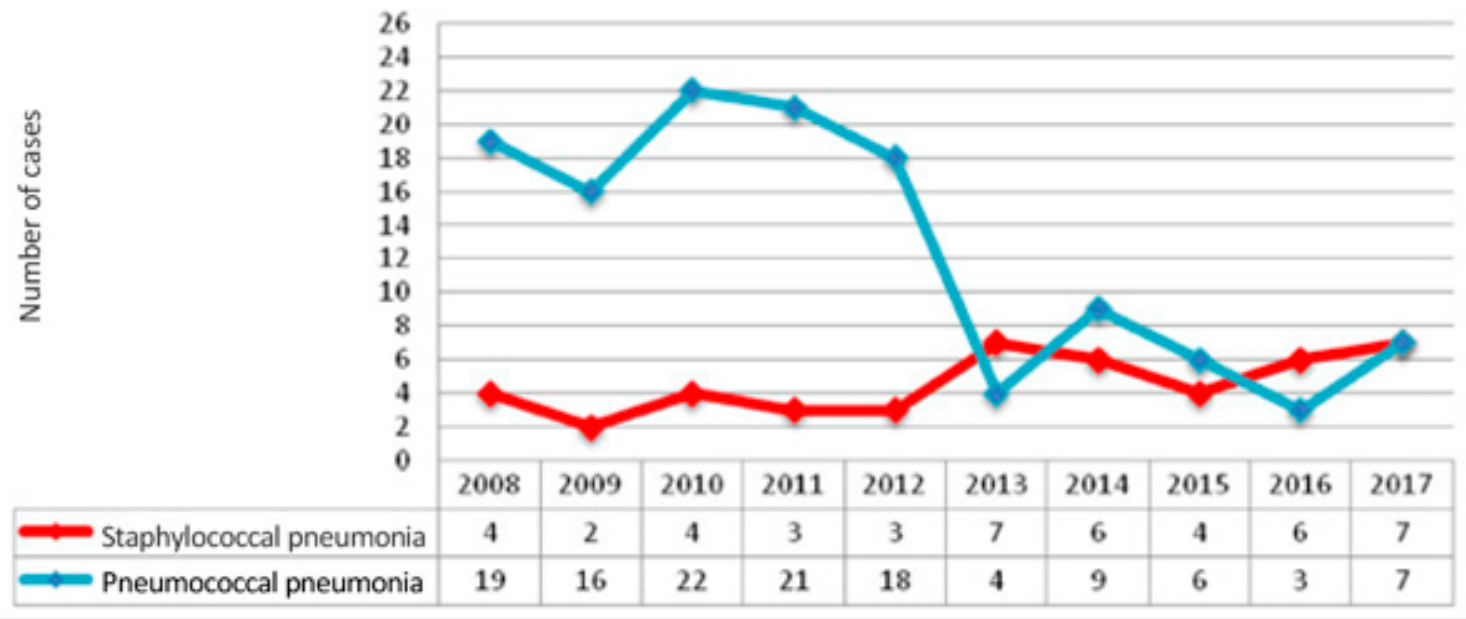

Hospital de Niños Víctor J. Vilela. 
Staphylococcal pneumonia was observed in almost half of sepsis or septic shock cases and, more commonly, in the form of necrotizing pneumonia or empyema, which more frequently required PICU admission, AMV, surgical debridement, and pleural drainage tube placement. It is worth noting that CA-MRSA pneumonia showed a 40-fold higher risk for complications due to pneumothorax, thus making evident its necrotizing characteristics and the procedures required for its management. ${ }^{8,18,19,24}$

In relation to the limitations of our study, it is worth noting that it was conducted at a referral children's hospital; therefore, findings should be extrapolated to other hospital care settings with caution. In addition, given the fact that the diagnosis of bacterial pneumonia without effusion is difficult because only $10-20 \%$ of blood cultures are positive, microorganism isolation in the absence of empyema or pleural effusion is usually low. The scarce number of deaths prevented us from assessing the impact of this infection on mortality.

One of the strengths of this study is its prospective nature for a planned data collection, which, among other contributions to the local context, allowed us to confirm the increased prevalence of CA-MRSA pneumonia in the past decade and outline the presentation characteristics that define its clinical treatment and management.

\section{CONCLUSIONS}

Out of all community-acquired SA pneumonia cases, $85 \%$ corresponded to CA-MRSA. The annual rate of CA-MRSA pneumonia increased from $4.9 / 10000$ to $10.1 / 10000$ discharges in the past 10 years. Compared to SP pneumonia, CA-MRSA pneumonia was more commonly accompanied by sepsis or septic shock, necrosis, and empyema, and required five times more surgical drainage, admission to the PICU, and AMV.

\section{REFERENCES}

1. Buckingham SC, McDougal LK, Cathey LD, Comeaux K, et al. Emergence of community-associated methicillinresistant Staphylococcus aureus at a Memphis, Tennessee Children's Hospital. Pediatr Infect Dis J. 2004; 23(7):619-24.

2. Miller LG, KaplanSL. Staphylococcus aureus: ACommunity Pathogen. Infect Dis Clin North Am. 2009; 23(1):35-52.

3. Kirkland EB, Adams BB. Methicillin-resistant Staphylococcus aureus and athletes. J Am Acad Dermatol. 2008; 59(3):494-502.

4. International Working Group on the Classification of Staphylococcal Cassette Chromosome Elements (IWG-SCC). Classification of staphylococcal cassette chromosome mec (SCCmec): Guidelines for reporting novel SCCmec elements. Antimicrob Agents Chemother. 2009; 53(12):4961-7.

5. Paganini H, Della Latta M, Muller Opet B, Ezcurra G, et al. Estudio multicéntrico sobre las infecciones pediátricas por Staphylococcus aureus meticilino-resistente provenientes de la comunidad en la Argentina. Arch Argent Pediatr. 2008; 106(5):397-403.

6. Frei CR, Miller ML, Lewis JS 2nd, Lawson KA, et al. Trimethoprim-sulfamethoxazole or clindamycin for community-associated MRSA (CA-MRSA) skin infections. J Am Board Fam Med. 2010; 23(6):714-9.

7. Morgan MS. Diagnosis and treatment of Panton-Valentine leukocidin (PVL)-associated staphylococcal pneumonia. Int J Antimicrob Agents. 2007; 30(4):289-96.

8. Rouzic N, Janvier F, Libert N, Javouhey E, et al. Prompt and successful toxin-targeting treatment of three patients with necrotizing pneumonia due to Staphylococcus aureus strains carrying the panton-valentine leukocidin genes. J Clin Microbiol. 2010; 48(5):1952-5.

9. Ensinck G, Casanueva E, Sguassero Y, Moyano M, et al. Infecciones de piel y partes blandas en pediatría: consenso sobre diagnóstico y tratamiento. Parte 2. Arch Argent Pediatr. 2014; 112(2):183-91.

10. Gentile A, Bakir J, Ensinck G, Cancellara A, et al. Staphylo$\operatorname{coccus} C$. meticilino resistente adquirido en la comunidad: hospitalización y riesgo de letalidad en 10 centros pediátricos de Argentina. Arch Argent Pediatr. 2018; 116(1):e47-53.

11. Haji T, Byrne A, Kovesi T. Trends in Pediatric Complicated Pneumonia in an Ontario Local Health Integration Net-

TABLE 4. Comparison of clinical presentation and course of staphylococcal and pneumococcal pneumonia. Hospital de Niños Victor J. Vilela, Rosario. 2008-2017 period

\begin{tabular}{lccc}
\hline & MRSA (46) & S. pneumoniae (125) & RR (95\% CI) \\
\hline Septic shock or sepsis & 19 & 7 & $7.38(3.32-16.38)$ \\
Pleuropulmonary suppuration & 44 & 52 & $2.30(1.85-2.86)$ \\
Necrotizing pneumonia or & & & \\
pneumonia with abscess formation & 22 & 12 & $4.98(2.69-9.23)$ \\
Pneumothorax & 16 & 1 & $43.48(5.93-318.65)$ \\
Surgery & 25 & 14 & $4.85(2.77-8.50)$ \\
Tubes & 41 & 48 & $2.14(1.70-2.70)$ \\
PICU & 30 & 19 & $4.29(2.70-6.83)$ \\
AMV & 15 & 7 & $5.82(2.54-13.37)$ \\
\hline
\end{tabular}

MRSA: methicillin-resistant Staphylococcus aureus; RR: relative risk; CI: confidence interval;

PICU: pediatric intensive care unit; AMV: assisted mechanical ventilation. 
work. Children (Basel). 2018; 5(3):36.

12. Rubinstein E, Kollef MH, Nathwani D. Pneumonia Caused by Methicillin $\square$ Resistant Staphylococcus aureus. Clin Infect Dis. 2008; 46(Suppl 5):S378-85.

13. Clinical and Laboratory Standards Institute (CLSI). Performance standards for antimicrobial susceptibility Testing. $30^{\text {th }}$ ed. CLSI supplement M100. Pennsylvania: CLSI;

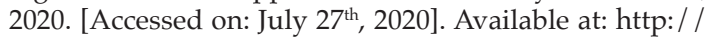
clsi.org/m100.

14. Wallin TR, Hern HG, Frazee BW. Community-Associated Methicillin-Resistant Staphylococcus aureus. Emerg Med Clin North Am. 2008; 26(2):431-55.

15. Iwamoto $\mathrm{M}, \mathrm{Mu} \mathrm{Y}$, Lynfield $\mathrm{R}$, Bulens $\mathrm{S}$, et al. Trends in invasive methicillin-resistant Staphylococcus aureus infections. Pediatrics. 2013; 132(4):e817-24.

16. Ensinck G, Ernst A, Lazarte G, Romagnoli A, et al. Infecciones por Staphylococcus aureus meticilino resistente adquirido en la comunidad: experiencia de 10 años en un hospital pediátrico de Rosario, Argentina. Arch Argent Pediatr. 2018; 116(2):119-25.

17. Paganini DH, Verdaguer V, Rodríguez AC, Della Latta P, et al. Infecciones causadas por Staphylococcus aureus resistentes a la meticilina en niños provenientes de la comunidad en niños de la Argentina. Arch Argent Pediatr. 2006; 104(4):295-300.

18. Carrillo-Marquez MA, Hulten KG, Hammerman W, Lamberth L, et al. Staphylococcus aureus pneumonia in children in the era of community-acquired methicillin-resistance at Texas Children's Hospital. Pediatr Infect Dis J. 2011; 30(7):545-50.

19. Li HT, Zhang TT, Huang J, Zhou YQ, et al. Factors associated with the outcome of life-threatening necrotizing pneumonia due to community-acquired Staphylococcus aureus in adult and adolescent patients. Respiration. 2011; 81(6):448-60.

20. Sola C, Paganini H, Egea AL, Moyano A, et al. Spread of epidemic MRSA-ST5-IV clone encoding PVL as a major cause of community onset staphylococcal infections in Argentinean children. PLoS One. 2012; 7(1):e30487.

21. Egea AL, Gagetti P, Lamberghini R, Faccone D, et al. New patterns of methicillin-resistant Staphylococcus aureus
(MRSA) clones, community-associated MRSA genotypes behave like healthcare-associated MRSAgenotypes within hospitals, Argentina. Int J Med Microbiol. 2014;304(8):108699.

22. See I, Wesson P, Gualandi N, Dumyati G, et al. Socioeconomic factors explain racial disparities in invasive community-associated methicillin-resistant staphylococcus aureus disease rates. Clin Infect Dis. 2017; 64(5):597-604.

23. Leggiadro RJ (ed.). Trends in Invasive Methicillin-resistant Staphylococcus aureus Infections. Current Abstracts. Pediatr Infect Dis J. 2014; 33(3):262.

24. Doudoulakakis AG, Bouras D, Drougka E, Kazantzi M, et al. Community-associated Staphylococcus aureus pneumonia among Greek children: epidemiology, molecular characteristics, treatment, and outcome. Eur J Clin Microbiol Infect Dis. 2016; 35(7):1177-85.

25. Adler A, Temper V, Block CS, Abramson N, et al. PantonValentine leukocidin-producing Staphylococcus aureus. Emerg Infect Dis. 2006; 12(11):1789-90.

26. Tristan A, Bes M, Meugnier H, Lina G, et al. Global distribution of Panton-Valentine leukocidin - positive methicillin-resistant Staphylococcus aureus, 2006. Emerg Infect Dis. 2007; 13(4):594:600.

27. Sicot N, Khanafer N, Meyssonnier V, Dumitrescu O, et al. Methicillin resistance is not a predictor of severity in community-acquired Staphylococcus aureus necrotizing pneumonia- results of a prospective observational study. Clin Microbiol Infect. 2013; 19(3):E142-8.

28. Schallcross LJ, Fragaszy E, Johnson AM, Hayward AC. The role of the Panton-Valentine leucocidin toxin in staphylococcal disease: A systematic review and meta-analysis. Lancet Infect Dis. 2013; 13(1):43-54.

29. Turner NA, Sharma-Kuinkel BK, Maskarinec SA, Eichenberger E, et al. Methicillin-resistant Staphylococcus aureus: an overview of basic and clinical research. Nat Rev Microbiol. 2019; 17(4):203-18.

30. Ves Losada J, Graziano AP, De Abreu M, Blanco M, et al. Infecciones graves por Staphylococcus aureus: características clínicas, sensibilidad antibiótica y uso de antimicrobianos. Serie de casos. Arch Argent Pediatr. 2014; 112(4):e152-5. 